\title{
THE RARE CASE PRESENTATION OF ASYMPTOMATIC CHEST WALL TUBERCULOSIS WITHOUT PULMONARY INVOLVEMENT IN PAEDIATRIC AGE GROUP.
}

KEY WORDS:

\section{Dr Kalpesh Onkar Patil*}

\section{Dr Satish Kulkarni Dr Kiran Maruti Gore}

Consultant Neonatal And Paediatric Laparoscopic Surgeon; Assistant Professor, Dept of Surgery, MIMER Medical College, Talegaon Dabhade, Pune.Dr.KP's Cherubs Child Clinic, Aundh, Pune. *Corresponding Author

Consultant Paediatrician And Neonatologist; Chiranjiv Pediatric Hospital, Chakan.Talegaon Dabhade, Pune.

\section{Consultant Pathologist; MD Pathology Christian Medical College,Vellore}

An Isolated chest wall tuberculosis (TB) is very rare form of presentation and represents $1 \%$ of all cases of osteoarticular tuberculosis. We report a case of 3 years old baby boy, vaccinated completely as per age and immunocompetent presented with asymptomatic chest wall swelling since last two years gradually increased to present size without any associated complaints and without any past history of any respiratory disease including pulmonary tuberculosis. Baby was treated with oral antibiotics for the same but did not respond to treatment symptomatically so referred for further treatment. On histopathology and microbiological study it showed tuberculous abscess.

\section{INTRODUCTION:}

Even today Tuberculosis (TB) is an important world health problem. Statistic in 2016 shows around 10.4 million cases of TB were documented of which $10 \%$ of cases were in paediatric age group younger than 18 years (1). In Mexico, a toll of 19,812 cases were reported in 2017 in general population, with an incidence rate of $17 / 100,000$ in habitants (2). Osteoarticular TB (OATB) represents $1 \%$ of the extrapulmonary TB in Mexico; however, rib cage TB has been documented only in few cases (1). In descending order, the commonest sites of chest wall tuberculosis are Sternum, ribs shafts, costochondral junctions and vertebras which may result from direct inoculation or haematogenous/lymphatic spread or as an extension of pulmonary disease. Diagnosis of an isolated chest wall tuberculosis is very difficult and require strong clinical acumen as it may resemble like pyogenic abscess. Herewith presenting a case of primary tuberculosis abscess in chest wall of a 3 years old baby boy vaccinated completely as per age, immunocompetent who presented with a swelling over chest gradually increasing in size over last 2 years and it was not associated with any history of respiratory complaints, significant family history or trauma.

\section{CASE REPORT}

A 3 years old baby boy referred with a painless oval swelling in the left lower thorax region. Swelling was almost pea sized $(1 \times 1 \mathrm{~cm})$ two years back which gradually increased to the present size of $10 \times 10 \mathrm{~cm}$. There was absolutely no history of any signs and symptoms of pulmonary TB like low grade fever, loss of appetite or loss of weight till date. All milestones have been achieved as per age. As swelling was very tiny in size initially and was not causing any problem to baby, parents neglected till the day it presented to current huge size. On examination, the baby was good built, playful, afebrile, and with normal pulse and blood pressure. Respiratory system examination was normal. Spine examination was normal. Local examination revealed a large solitary lesion over left lower thoracic region of size $10 \times 10 \mathrm{~cm}$ which was firm, nontender, euthermic, partially mobile with well-defined margins and seems to be not attached to underlying bony structures (Fig. 1). There was no involvement of the regional lymph nodes. Since the lesion started growing over last few months, it was treated with some antibiotic course by nearby paediatrician but could not see any clinical improvement so referred to us for further treatment. No any medical investigations were done. On haematological investigations, all reports including hemogram, liver and renal functions were within reference ranges. Serology for HIV was nonreactive. Xray chest did not show any abnormality. Loco regional ultrasonography showed huge cystic cavity extending inside between the intercostal space touching to pleura with no any evidence of pleural effusion or any other abnormality. Contrast computed tomography images of chest (Fig.2) confirmed the sonography findings with no evidence of erosion of sternum, vertebras and ribs. Lung parenchyma on either side along with the rib cage and spine was normal entirely. Patient was planned for wide excision of cyst under anaesthesia. After the complete pre anaesthesia check up and parental consent baby was posted for surgical excision of mass. Intra operative swelling was dissected well from all side but suddenly it got burst out during dissection and very thick milky white pus (around $90 \mathrm{ml}$ ) drained out. Complete cavity wall was scooped, rib beneath cyst was partially shaved off and specimen sent for histopathology. Pus was collected and sent for microbiology tests. After wound was closed in layers keeping a drain in situ. Post operative recovery was uneventful. The drained pus material was sent to the microbiology laboratory for pyogenic culture and Ziehl Neelsen (ZN) staining. There was no growth of any pyogenic organism after $48 \mathrm{hrs}$ of incubation but acid fast bacilli (AFB) were seen in ZN stain (Fig 3). Sample grew Mycobacterium tuberculosis on Lowenstein Jensen medium after one week of incubation. In line probe assay, the isolate was identified as M.tuberculosis and patient was put on category 1 antitubercular treatment as per culture and sensitivity report consisting of an intensive phase of $\mathrm{H}, \mathrm{R}, \mathrm{Z}$, and $\mathrm{E}$ administered under direct supervision thrice weekly on alternate days for 2 months, followed by a continuation phase of $\mathrm{H}$ and $\mathrm{R}$ thrice weekly on alternate days for 4 months. On follow up wound was healed completely with no recurrence and fresh complaints.

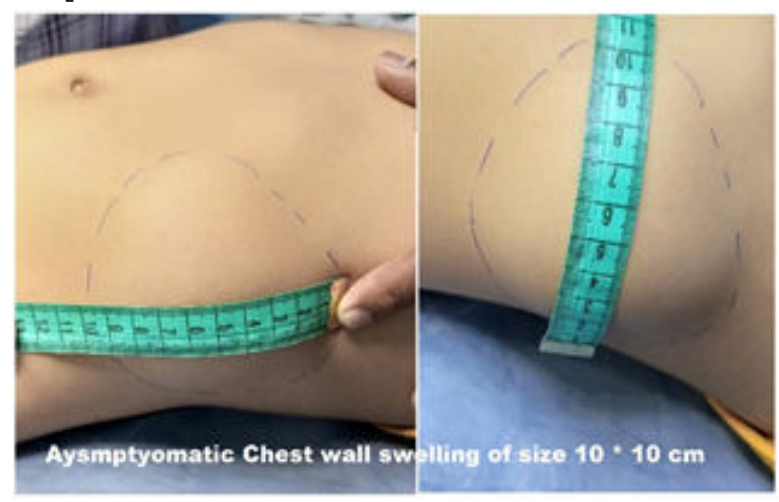

Figure 1: Clinical examination of swelling over left side lower thorax 


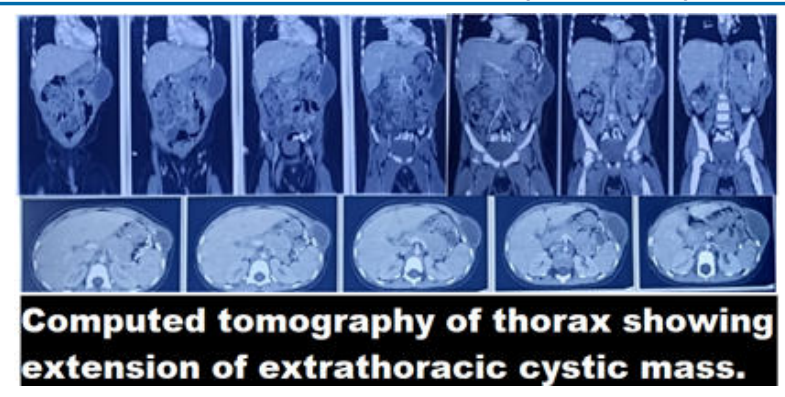

7. TB INDIA 2014 Revised National TB Control Programme Annual status report," Central B Division, Directorate General Health Services, Ministry of Health and FamilyWelfare, 2014

Figure 2. CT scan thorax showing the extension of extra thoracic cystic mass.

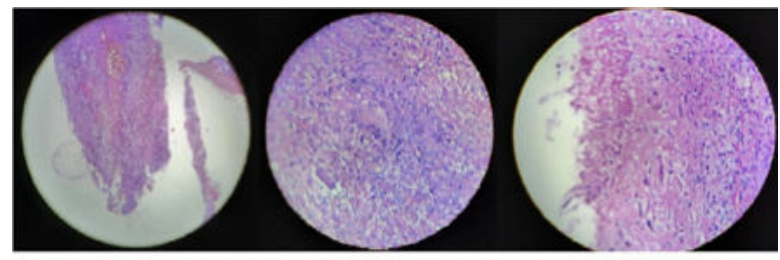

Histopathological and ZN staining slides showing acid fast bacilli

\section{Figure 3. Histopathology slides showing acid fast bacilli}

\section{DISCUSSION}

There are different mechanisms described of an isolated chest wall abscess such as direct extension from plural or pulmonary tuberculosis, haematological dissemination of a dormant tuberculous focus and direct extension from chest wall lymphatics $(3,4)$.

An isolated primary tuberculous chest wall swelling is rare and diagnosis in most of the cases is demanding and effortful because the lesions grossly simulate pyogenic abscess or tumour and do not respond to conventional therapeutic interventions. Chest wall abscess usually occurs as a solitary cystic lesion, most frequently at the margins of the sternum and in the shafts of the ribs (5). Even though the aspirated pus shows acid fast bacilli growth showing tuberculous aetiology of the lesion, the site of lesion is also mandatory as mycobacteria other than tuberculosis are more frequently associated with skin lesions also and are the most significant cause of the treatment failure of anti-tubercular treatment (6). Though Revised National Tuberculosis Control Programme (RNTCP) recommends a standard 6 months regimen with 2 months of intensive phase (HRZE drugs) and 4 months of continuation phase (HR) (7), Still there is controversy remained regarding the duration of treatment of chest wall tuberculosis; few reports suggest good response with antitubercular drugs only, while others suggest wide surgical debridement along with antitubercular drugs. Primary tubercular involvement of extrapulmonary site like chest wall abscess is extremely rare and needs high clinical acumen; also, identification of extrapulmonary isolates would be absolutely essential for instituting right therapeutic intervention.

\section{REFERENCES}

1. Rafiqi K, Yousri B, Arihi M, Bjitro C, Aboumaarouf M, El Andaloussi M, 2013. Unusual locations of osteoarticular tuberculosis in children: a report of 12 cases. Orthop Traumatol Surg Res 99:347-351

2. National Epidemiological Surveillance System (SINAVE) of Mexico, 2015. Epidemiological Profile of Tuberculosis in Mexico [Perfil Epidemiolo' gico de la Tuberculosis enMe' xico].Health Ministry, SINAVE

3. M. Sakuraba, Y. Sagara, and H. Komatsu, "Surgical treatment of tuberculous abscess in the chest wall," Annals of Thoracic Surgery, vol. 79, no. 3, pp. 964-967,2005.

4. K.D. Cho, D. G. Cho, M. S. Jo, M. I. Ahn, and C. B. Park, "Current surgical therapy for patients with tuberculous abscess of the chest wall," Annals of Thoracic Surgery, vol.81,no. 4,pp. 1220-1226,2006.

5. H. E. Burke, "The pathogenesis of certain forms of extrapulmonary tuberculosis; spontaneous cold abscesses of the chest wall and Pott's disease," The American Review of Tuberculosis, vol. 62, no. 1 B, pp. 48-67, 1950

6. C. Piersimoni and C. Scarparo, "Extrapulmonary infections associated with nontuberculous mycobacteria in immunocompetent persons," Emerging Infectious Diseases, vol. 15, no.9, pp. 1351-1358,2009 